\title{
Morphology and evolution of umbral dots and their substructures
}

\author{
M. Sobotka ${ }^{1}$ and K. G. Puschmann ${ }^{2}$
}

\author{
1 Astronomical Institute, Academy of Sciences of the Czech Republic (v.v.i.), Fričova 298, 25165 Ondřejov, Czech Republic \\ e-mail: msobotka@asu.cas.cz \\ 2 Instituto de Astrofísica de Canarias, vía Láctea S/N, 38200 La Laguna, Tenerife, Spain \\ e-mail: kgp@iac.es
}

Received 21 April 2009 / Accepted 5 June 2009

\begin{abstract}
Context. Substructures - dark lanes and tails - of umbral dots (UDs) were predicted by numerical simulations of magnetoconvection and have been detected later in some observations.

Aims. To provide constraints for realistic theoretical models of sunspot umbrae, we describe the observed properties and evolutionary characteristics of UDs (including their substructure) and of other umbral structures.

Methods. We analyse a $6 \mathrm{~h} 23$ min time series of broadband images of a large umbra in the active region NOAA 10634, acquired with the 1-m Swedish Solar Telescope, in the wavelength band around $602 \mathrm{~nm}$. A $43 \mathrm{~min}$ part of this series was reconstructed with the MFBD method, reaching a spatial resolution of 0 ! $^{\prime} 14$. With the help of image segmentation, feature tracking, and local correlation tracking, we measured brightness, size, lifetime, and horizontal velocities of various umbral structures.

Results. Large structures in the umbra - strong and faint light bridges (LBs) and an extended penumbral filament - evolve on time scales of hours. Most $(90 \%)$ of UDs and bright point-like features in faint LBs split and merge, and their median lifetimes are 3.5 or $5.7 \mathrm{~min}$, depending on whether the split or merge event is considered as the end of their life. Both UDs and features in faint LBs that do not split or merge are clearly smaller $\left(0{ }^{\prime} \cdot 15\right)$ than the average size $\left(0{ }^{\prime} 17\right)$ of all features. Horizontal motions of umbral bright small-scale features are directed either into the umbra or along faint LBs with mean horizontal velocities of $0.34 \mathrm{~km} \mathrm{~s}^{-1}$. Features faster than $0.4 \mathrm{~km} \mathrm{~s}^{-1}$ appear mostly at the periphery of the umbra. The motion of peripheral UDs (PUDs) seems to be the continuation of the motion of penumbral grains (PGs). The intensity of dark lanes, measured in four bright central UDs (CUDs), is by a factor 0.8 lower than the peak intensity of CUDs. The width of dark lanes is probably less than the resolution limit 0 ' $^{\prime} 14$. The characteristic time of substructure changes of UDs is $\sim 4$ min. We observe narrow ( $\left.0{ }^{\prime}{ }^{\prime} 14\right)$ bright and dark filaments connected with PUDs. The bright filaments are $0.06 I_{\mathrm{ph}}$ brighter than the dark ones. Usually one dark and two bright filaments form a 0' 4 wide tail attached to one PUD, resembling a short dark-cored penumbral filament.

Conclusions. Our results indicate the similarity between PUDs and PGs located at the tips of bright penumbral filaments. The features seen in numerical MHD simulations are consistent with our observations of dark lanes in CUDs and tails attached to PUDs.
\end{abstract}

Key words. sunspots - Sun: photosphere

\section{Introduction}

The study of fine structure in sunspot umbrae is crucial to understanding the interactions of plasma with strong magnetic fields and the physical structure of sunspots. Currently, thanks to new ground-based and space instruments, image reconstruction methods, and MHD simulations, our knowledge of fine-structure elements is showing considerable progress. This concerns umbral dots (UDs), the small isolated bright features observed in the umbra, and light bridges (LBs), the elongated bright features crossing the umbra. At the umbra-penumbra boundary, penumbral grains (PGs) located at the tips of bright penumbral filaments sometimes protrude deep into the umbra. Usually, UDs are divided into two groups, peripheral umbral dots (PUDs) and central umbral dots (CUDs), according to their position inside the umbra (Grossmann-Doerth et al. 1986). For definitions and basic characteristics of the sunspot fine structures, we refer to the monograph by Thomas \& Weiss (2008).

Two basic approaches have been proposed to explain the nature of UDs. According to Choudhuri (1986), UDs are columns of hot field-free gas intruding into a cluster of thin magnetic flux tubes that form the magnetic structure of a sunspot (Parker 1979). Another approach, based on the magnetoconvection in a monolithic thick flux tube, has been proposed by Weiss et al. (1990). In their model, UDs are hot convective plumes that overshoot to the sunspot's photosphere. This approach was extensively elaborated by means of 3D numerical simulations of magnetoconvection. Recently, Schüssler \& Vögler (2006) have shown that the energy transport in the vertical magnetic field of the umbra is dominated by nonstationary narrow plumes of rising hot plasma with adjacent downflows. The magnetic field is strongly reduced in the upper layers of the plumes. At the visible surface $(\tau=1)$, the plumes are seen as bright features with a typical size of $200-300 \mathrm{~km}$ and lifetime of the order of $30 \mathrm{~min}$, resembling UDs. Most of the simulated UDs have an elongated shape with a central dark lane, some larger UDs show a threefold dark lane. The dark lanes are a consequence of enhanced density and opacity at the top of the plume, so that the continuum formation layer is shifted upwards to cooler layers of the atmosphere. Rempel et al. (2009) extended the simulation, introducing a 2D (slab) magnetic field fanning out with height into a 3D convective media. They obtained a sunspot-like structure surrounded by photospheric granulation. In the umbra of the "sunspot", UDs similar to those simulated by Schüssler \& Vögler (2006) were present. At the periphery of the umbra in a more inclined magnetic field (with respect to the vertical), UDs 
appeared with two attached bright narrow tails pointing toward the penumbra. Even farther from the centre of the umbra structures developed resembling PGs followed by dark-cored penumbral filaments (see Fig. 1 of Rempel et al. 2009).

The most recent extensive imaging observations of UDs have been published by Riethmüller et al. (2008b). They observed thousands of UDs in a 106 min long time series of images acquired in the TiO band head $(705.7 \mathrm{~nm})$ with the $1-\mathrm{m}$ Swedish Solar Telescope (SST). Applying a feature tracking algorithm, they measured sizes, brightnesses, lifetimes, trajectories, and horizontal velocities. The majority of UDs was spatially resolved with typical diameter of 0.31 . A difference in the evolution of brightness is found between CUDs and PUDs, pointing to a different origin of these types of UDs. The behaviour of CUDs shows better agreement with the simulations of Schüssler \& Vögler (2006) than that of PUDs. A new subset of PUDs, mobile UDs with travel distances over $1^{\prime \prime}$, was introduced. Mobile UDs are born close to the umbra-penumbra boundary, they are brighter, live longer, and move faster $\left(680 \mathrm{~m} \mathrm{~s}^{-1}\right)$ than the rest of UDs $\left(410 \mathrm{~m} \mathrm{~s}^{-1}\right)$. Properties of UDs were also recently studied by Kitai et al. (2007) in three series of broad-band images acquired by Hinode/SOT.

Recent spectroscopic and spectropolarimetric observations have provided new information about line-of-sight (LOS) velocities and magnetic fields. Analysing Dopplergrams obtained at the Dunn Solar Telescope, Bharti et al. (2007a) found upflows of $400 \mathrm{~m} \mathrm{~s}^{-1}$ surrounded by downflows of $300 \mathrm{~m} \mathrm{~s}^{-1}$ in large (0.'5) UDs. The discovery of downflows strongly supports the magnetoconvective origin of UDs. Full Stokes spectra of 21 CUDs and 30 PUDs, observed by Hinode/SOT, were inverted by Riethmüller et al. (2008a). At the continuum level $(\tau=1)$, they find a magnetic field weakening of $480 \mathrm{G}$ in CUDs and $510 \mathrm{G}$ in PUDs. No significant LOS velocities have been detected in CUDs, but PUDs are connected with upflows of $800 \mathrm{~m} \mathrm{~s}^{-1}$. The physical differences between CUDs and PUDs have been confirmed by Sobotka \& Jurčák (2009), using a 3 h long time series of full-Stokes spectral scans obtained with Hinode/SOT. In the range of optical depths $0.3<\tau<0.6$, CUDs do not show any excess of LOS velocity and magnetic field inclination with respect to the surrounding umbra, while upflows of $400 \mathrm{~m} \mathrm{~s}^{-1}$ and a more horizontal magnetic field are observed in PUDs. It seems that PUDs are more like PGs than CUDs. Both PUDs and PGs appear in regions with a weaker and more horizontal magnetic field and their formation height reaches the low photosphere, while CUDs appear in regions with stronger and more vertical magnetic fields, and they are formed too deep to detect upflows and changes in magnetic field inclination.

Dark lanes in UDs, predicted in the simulations by Schüssler \& Vögler (2006), have been observed by Bharti et al. (2007b) in large $\left(>1^{\prime \prime}\right)$ bright UD-like features seen in Hinode/SOT G-band images with diffraction-limited spatial resolution of 0.22 . After inverting a nearly contemporaneous Hinode/SOT spectropolarimetric scan, Bharti et al. (2009) conclude that the dark lanes correspond to a temperature deficit located above $\tau=0.1$, which, together with observed upflows and magnetic field reduction in UDs, supports the results of theoretical simulations. In G-band images acquired with the Dunn Solar Telescope, Rimmele (2008) observed dark dots or lanes within bright UDs. Their size is typically close to the diffraction limit of $0{ }^{\prime} 12$. Close to the umbral border, Rimmele finds elongated dark structures associated with bright PUDs moving into the umbra. These dark structures have the appearance of a short dark tail pointing toward the penumbra, and they resemble dark-cored filaments originating at PGs.
In this paper we describe the fine structure of a large and dark umbra, observed for more than $6 \mathrm{~h}$ with the spatial resolution $0{ }^{\prime} 14$ in a large number of broad-band images. First we describe the observed long-term characteristics and evolution of the umbra, LBs, and long penumbral filaments extending into the umbra. Then we centre on some statistical properties of umbral features. Some preliminary results were published previously in two conference papers (Sobotka \& Puschmann 2007; Sobotka et al. 2008, hereafter Papers I and II). Finally we focus on the substructures of UDs - dark lanes in CUDs and fine bright and dark filaments composing tails associated with PUDs.

\section{Observations and data processing}

The large leading sunspot in NOAA 10634 was observed on 18 June 2004 from 07:43 to 15:30 UT with the 1-m SST, La Palma (Scharmer et al. 2003), equipped with adaptive optics. The spot, located near the disk centre (N13, E12), was growing to the maximum phase of evolution. The broad-band images were acquired simultaneously in three wavelength bands: blue $(450.8 \pm 0.5) \mathrm{nm}$, red $(602.0 \pm 1.3) \mathrm{nm}$, and G-band $(430.89 \pm 0.6) \mathrm{nm}$. Exposure times were $11-14 \mathrm{~ms}$ and the pixel size was $0{ }^{\prime} 0405 \times 00^{\prime} 0405$. The images were acquired in the frame-selection mode during most of the observing period. The selection interval was $20 \mathrm{~s}$ and the average time difference between two frames (including the time necessary to store the images) is $20.1 \mathrm{~s}$. From 08:40 to 09:30 UT, all frames (cadence 4-5 s $\mathrm{s}^{-1}$ ) were stored to be used later for image reconstruction by MFBD (the special treatment of these data is described in this section later on). A post-facto frame selection was applied to these frames to obtain a homogeneous time series during the full observing period with a temporal resolution of $20.1 \mathrm{~s}$.

After dark- and flatfield corrections, the intensities in each image were normalised with respect to the average intensity $I_{\mathrm{ph}}$ of a quiet granulation area near the sunspot to compensate for changes of transparency and/or exposure time. Photospheric and penumbral stray light, which contaminates the umbra due to the scattering in the terrestrial atmosphere and in the instrument, was eliminated using the method by Martínez Pillet (1992). Parameters of the scattering were determined for each wavelength from the shapes of photometric profiles across the solar limb. Images of the limb were recorded immediately after the observation. The level of stray light, expected to originate mostly in the instrument, was $8.5 \%$ in blue, $6.5 \%$ in red, and $8.7 \%$ in G-band. A deconvolution of the instrumental profile of the diffraction-limited 1-m telescope and a simultaneous noise filtering was applied to the frames of the series, using Wiener filters with noise suppression starting at 0.'11 (blue), 0.'14 (red), and 0.13 (G-band). Regarding the correction of wavefront aberrations done by the adaptive optics, these values characterise the spatial resolution in the best frames. Then, the image rotation was compensated and the frames were aligned and de-stretched. Finally, a subsonic filter with a cutoff at $4 \mathrm{~km} \mathrm{~s}^{-1}$ was applied to remove a residual jitter due to the seeing. The beginnings and ends of the series were removed because of insufficient image quality and apodisation effect. The resulting three time series in blue, red, and G-band wavelength ranges span from 08:05 to 14:28 UT, i.e., 6 h 23 min. The G-band series is not suitable for the study of umbral structures, because the darkest parts of the umbra are underexposed. The red continuum series with the best signal-to-noise ratio $(S / N=200)$ was selected for further analysis.

As mentioned above, a part of the time series (from 08:40 to $09: 30 \mathrm{UT}$ ) has been treated with special care. During this 
50 min time period the seeing was not extremely good but very stable, offering a good opportunity for an efficient image restoration. We stored all the images (a total of 12000 frames in each blue and red band) acquired at a frame rate of 4-5 images $\mathrm{s}^{-1}$. We applied the post-facto multi-frame blind deconvolution (MFBD) image restoration algorithm developed and implemented by Löfdahl (2002) on the recorded dark- and flatfield corrected images. This reconstruction method uses multiple frames to retrieve the residual aberrations affecting the images after the partial correction performed by the adaptive optics and thus further improves the image quality. In the present case we combined sets of 50 sequential images to obtain single restored ones. Thus, the resulting sequences of the red and blue bands last $50 \mathrm{~min}$ and consist of 236 reconstructed images with a temporal cadence of $12.6 \mathrm{~s}$ each. This sampling interval has been chosen such that the amount of images grouped for the restoration is as large as possible without losing important physical information about the temporal evolution of our science target. Moreover, the reconstruction is performed in small portions of the images where one can consider a common characteristic aberration (isoplanatic patches). Overlapping patches of $128 \times 128$ pix $\left(5{ }^{\prime \prime} 184 \times 5{ }^{\prime \prime} 184\right)$ have been extracted such that at the end of their restoration the whole field of view (FOV) can be recovered by mosaicking them without any discontinuity. Proceeding in this way, the homogeneity and image quality along the time series and in the whole FOV can be improved up to close to the diffraction limit of the telescope. The resulting reconstructed time series have been treated with the standard procedures, i.e., image de-rotation, image alignment, and subsonic filtering (again with a cutoff of $4 \mathrm{~km} \mathrm{~s}^{-1}$ ). Some frames of these series were lost due to apodisation, so that the resulting 43 min long blue- and redband series contain 204 frames each and span from 08:43 to 09:26 UT.

The horizontal motions of umbral features were studied using the methods of local correlation tracking (LCT, November \& Simon 1988) and feature tracking. LCT provides a timeaveraged horizontal velocity field of all structures in the FOV. The FWHM of the Gaussian tracking window was set to 0.32 and the temporal integration was made over $1 \mathrm{~h}$ and $2 \mathrm{~h}$. There was no substantial difference between the velocity fields obtained with these two integration times. The feature tracking provides time records of positions, intensities, and sizes for each individual feature. We used the algorithm developed by Sobotka et al. (1997) that has recently been improved by Hamedivafa (2008). This algorithm makes it possible to distinguish between features that split into two or more parts, merge with another feature, or preserve their integrity during their life.

\section{Results}

\subsection{Long-term evolution of umbral structures}

The evolution of the umbra was inspected visually in the movie composed of the red-band images taken from 08:05 to 14:28 UT. One of the images, taken at 12:21 UT, is shown in Fig. 1. The umbra was very stable during our observation. Its area $A=345 \operatorname{arcsec}^{2}$, corresponding to the effective diameter $d_{\mathrm{eff}}=$ $\sqrt{4 A / \pi}=21^{\prime \prime}$, was constant within this period. The area was defined by the intensity threshold $0.5 I_{\mathrm{ph}}$. The observed umbra is very dark. After stray-light correction, the minimum intensity in the umbra is $0.09 I_{\mathrm{ph}}\left(0.05 I_{\mathrm{ph}}\right.$ in the blue band), remaining constant with time. The whole umbra shows a slowly evolving grainy and/or filamentary low-contrast "background" pattern with rms fluctuations of only 0.01-0.02 $I_{\mathrm{ph}}$ (Paper I).

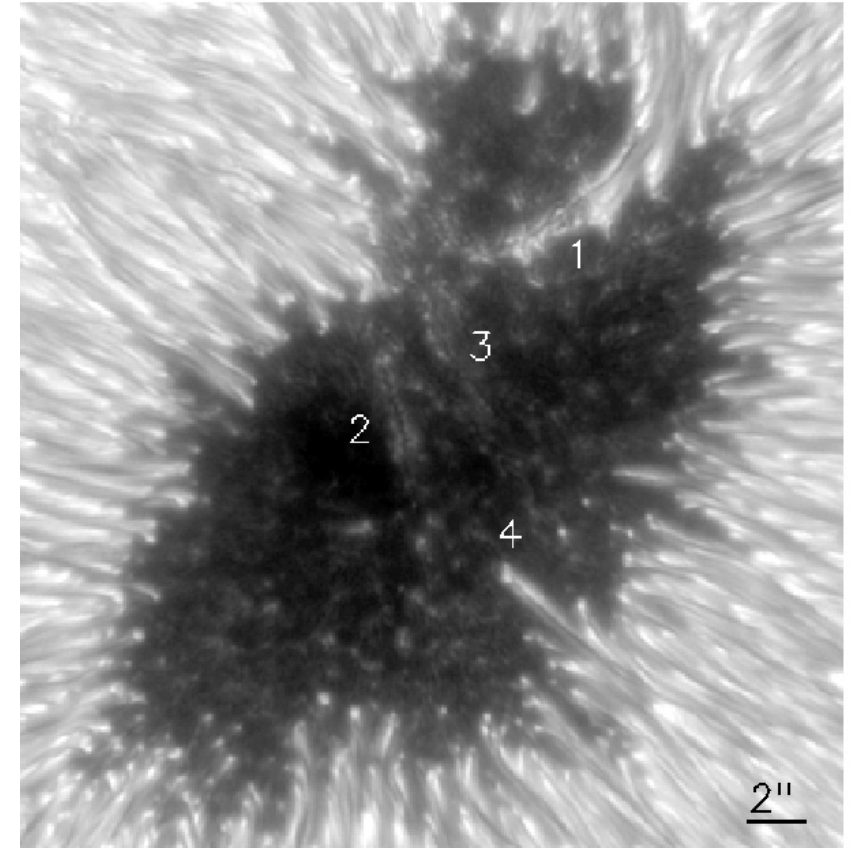

Fig. 1. Red-band image of the umbra taken at 12:21 UT. 1 - Strong light bridge; 2, 3 - faint light bridges; 4 - long penumbral filament.

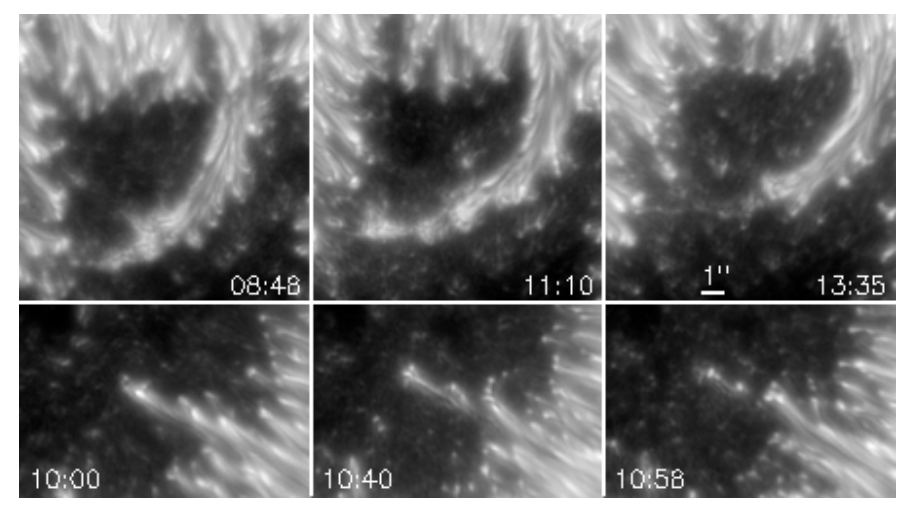

Fig. 2. Snapshots of evolution of the strong light bridge marked as " 1 " in Fig. 1 (top row) and of the long penumbral filament "4" (bottom row).

Bright inward-moving UDs travel in this background pattern. Even a dark nucleus, the less intense part of the umbra, where the strongest magnetic field is expected, shows a very faint grainy and filamentary structure. Identical patterns were detected in the red and blue bands, proving that we observe real structures, so the umbral background does not have the diffuse character expected in the past.

A strong LB, marked as " 1 " in Fig. 1, divided the umbra into two umbral cores. One part of the bridge (top right in Fig. 1) was composed of extended penumbral filaments with dark cores, while the other part had a granular structure with a central dark lane. The dark lane was not connected to any of the dark cores in the penumbral filaments. After $3 \mathrm{~h} 5 \mathrm{~min}$ of the observation (at 11:10 UT), the granular part split into several fragments that weakened gradually, until the minimum brightness was reached at 13:35 UT (see snapshots of evolution in Fig. 2, top row). Then the granular part started to recover. Bright grains moved along the filamentary part of the LB toward the granular one with typical velocities of $0.8-1 \mathrm{~km} \mathrm{~s}^{-1}$ (LCT) all the time.

The long penumbral filament " 4 ", extending deep into the umbra, passed several evolutionary phases. It had been present since the beginning of the observation. Starting from 08:22 UT, 
its length increased with an average speed of $0.6 \mathrm{~km} \mathrm{~s}^{-1}$ during $40 \mathrm{~min}$. After that growing phase, it remained stable for 50 min and then it started to grow again. The second growing phase took approximately $30 \mathrm{~min}$. In the next $20 \mathrm{~min}$ the filament separated from the penumbra and then, after a $30 \mathrm{~min}$ long decay phase, it disappeared at 11:10 UT. The bright head of the filament converted into three PUDs that moved toward LB 2. Three snapshots displayed in the bottom row of Fig. 2 show this filament in the second growing phase, during its separation from the penumbra, and in the decay phase. At the same time (11:10 UT), a new long penumbral filament developed at the distance of $1 . .3$ from the original one. It showed the same phases of evolution at very similar time scales - growth $\left(50 \mathrm{~min}\right.$, speed $\left.0.6 \mathrm{~km} \mathrm{~s}^{-1}\right)$, stability (50 min), second growth (30 min), separation (20 min), and decay (30 min). It disappeared at 14:11 UT and its total lifetime was $3 \mathrm{~h}$. During the existence of both filaments, bright PGs moved along them with speeds up to $1.2 \mathrm{~km} \mathrm{~s}^{-1}$ (LCT) and many PUDs, moving toward LB 2, detached from the heads of the filaments.

Faint granular LBs "2" and "3" (Fig. 1) started to form at 10:05 and 09:15 UT, respectively. They developed into well-distinguishable structures with central dark lanes around 11:45 UT. During the formation period, the motion $(0.2-$ $0.3 \mathrm{~km} \mathrm{~s}^{-1}$, LCT) of bright features along LB 3 and along the upper part of LB 2 was oriented into the umbra. In the lower part of LB 2, an opposite motion toward the penumbra $\left(0.3 \mathrm{~km} \mathrm{~s}^{-1}\right)$ was seen. When the formation was finished, the opposite motion of $0.4 \mathrm{~km} \mathrm{~s}^{-1}$ prevailed in LB 2 (see Paper I). Such opposite motions along two adjacent LBs were also observed by Rimmele (2008). This LB started to decay at 13:55 UT, while LB 3 remained stable till the end of the observation. The opposite motion of bright features in LB 2 might be related to a horizontal velocity field connected with two long penumbral filaments that developed subsequently at the position "4" in Fig. 1. An example of an LCT map of horizontal velocities in the umbra is shown in Fig. 3. This map is calculated for the period from 12:15 to 14:12 UT.

Bright inward-moving PUDs are often followed by faint bright filaments, resembling penumbral filaments attached to PGs, and/or by dark "wakes", resembling dark cores of penumbral filaments (Paper I). Similar structures were reported independently by Rimmele (2008). The LCT flow map (see Fig. 3) shows that the horizontal motion of PUDs $\left(0.5 \mathrm{~km} \mathrm{~s}^{-1}\right)$ seems to be the continuation of the inward-directed motion field of PGs in the inner penumbra.

\subsection{Statistical properties of umbral features}

In the following we focus on some detailed statistical results obtained for the best part of the unreconstructed series in the red band, taken in the frame-selection mode from 12:15 to 14:12 UT. Movies covering $90 \mathrm{~min}$ of the time series from 12:28 to 13:58 UT can be found at http://www . asu.cas.cz/ sdsa/ gallery-astropictures.html.

The feature-tracking technique was applied to this $117 \mathrm{~min}$ long unreconstructed time series. The bright features were separated from the background using the low-noise curvature determination algorithm (Hamedivafa 2008). Only features (UDs and dot-like features in faint LBs) brighter by $0.04 I_{\mathrm{ph}}$ than the surrounding umbra, larger than 0 .' 14 (resolution limit), and living longer than 2 min were taken into account. The resulting sample contains 3099 UDs and 1357 dot-like features in LBs.

The majority $(90 \%)$ of observed features split or merge with other features (see Paper II), thus it is necessary to take into

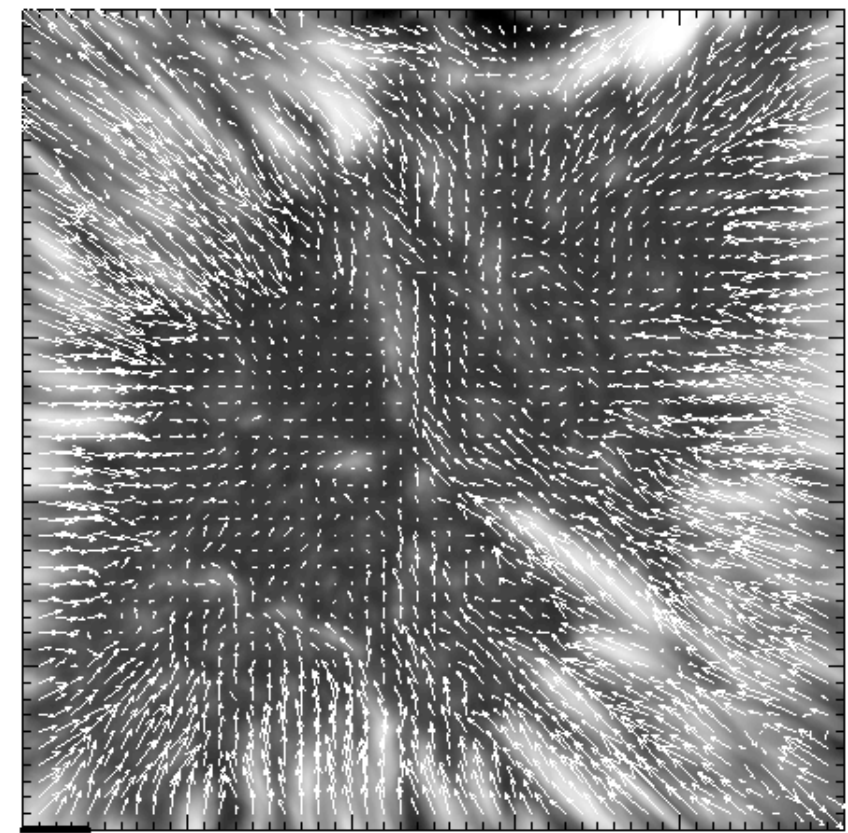

Fig. 3. LCT map of horizontal velocities, calculated with the tracking window of 0.32 and averaged over a period of $117 \mathrm{~min}$, the best part of the unreconstructed series in the red band, taken in the frame-selection mode from 12:15 to 14:12 UT. The length of the black horizontal bar at the bottom left corner represents $1 \mathrm{~km} \mathrm{~s}^{-1}$.

account these effects. One possibility is to finish the time record of a feature when it splits or merges. In this case, the mean (median) lifetimes of UDs are $4.5 \mathrm{~min}(3.7 \mathrm{~min}$ ) and the mean (median) lifetimes of the features in faint LBs are $4.4 \mathrm{~min}$ (3.4 min). On the other hand, split and merge events caused by seeing effects and random coincidences may spuriously shorten the lifetime of many UDs. The used feature tracking algorithm makes it possible to connect the records of features before and after the split/merge event according to the following rules: (a) if a feature splits, its successor is the brightest fragment; (b) if two or more features merge, the resulting feature is connected to its longest-lived predecessor. In this case, the mean (median) lifetimes of UDs are $9.1 \mathrm{~min}(5.7 \mathrm{~min})$ and the mean (median) lifetimes of the features in faint LBs are $9.8 \mathrm{~min}(5.7 \mathrm{~min})$. Independently of the applied method, the histograms of lifetimes show a monotonous decrease from shortest to longest lifetimes as reported previously by Sobotka et al. (1997) and Riethmüller et al. (2008b).

Both UDs and features in faint LBs 2 and 3 that do not split or merge are smaller than the average size of all features (Paper II). Histograms of effective diameters calculated from time-averaged areas of all 3099 UDs and 1357 bright features in LBs and of 337 UDs and 114 bright features in LBs that did not split or merge are plotted in Fig. 4, top row. It can be seen that UDs and bright features in LBs do not differ in size. However, the non-split/merge features have narrower range of sizes and smaller mean size $\left(0{ }^{\prime} 15\right)$ than all features in the sample (with the mean size of $\left.0{ }^{\prime} 17\right)$. Larger diameters of splitting or merging features suggest that these features may be composed to a large extent of small unresolved objects.

The feature tracking was also used to measure the horizontal velocities of small-scale umbral features. Motions of UDs are mostly directed into the umbra, while the features in faint LBs 2 and 3 move predominantly along the bridges. Histograms of time-averaged velocity magnitudes are plotted in Fig. 4, bottom 

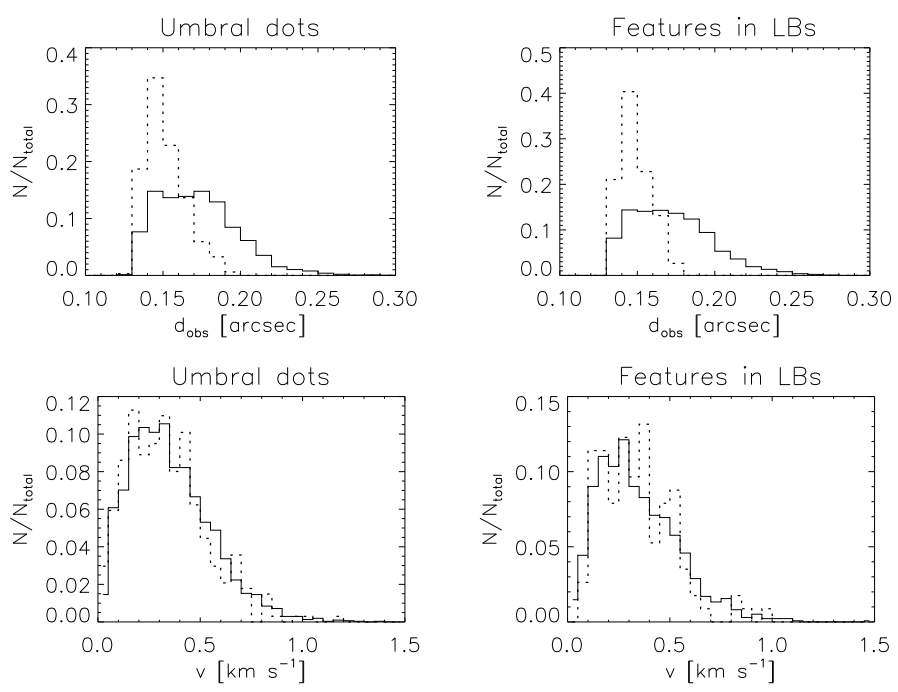

Fig. 4. Histograms of time-averaged effective diameters (top) and timeaveraged horizontal velocity magnitudes (bottom), derived from feature tracking. Dotted line - non-split/merge features, solid line - all features.
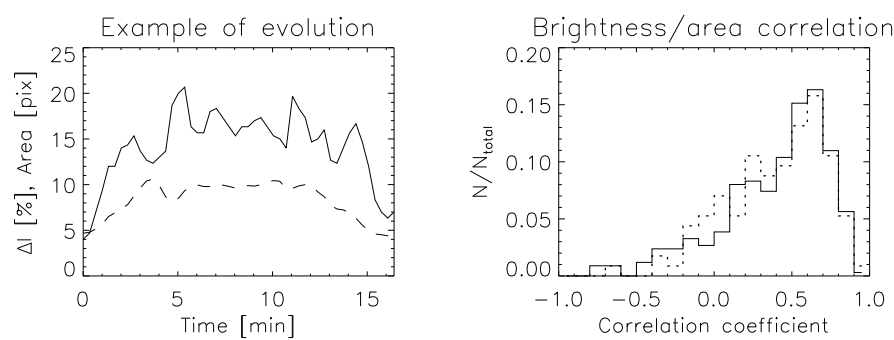

Fig. 5. Left - example of area (solid) and brightness (dashed) evolution of a central umbral dot. Right - histograms of the correlation between brightness and area during the evolution of individual non-split/merge features. Solid line - umbral dots, dotted line - bright features in faint light bridges.

row. We see that the velocity magnitudes are similar for UDs and features in LBs and do not depend on split or merge of the features. All types of features show a mean velocity around $0.34 \mathrm{~km} \mathrm{~s}^{-1}$. A map of horizontal velocity vectors (not shown here) indicates that features faster than $0.4 \mathrm{~km} \mathrm{~s}^{-1}$ appear mostly at the periphery of the umbra and in faint LBs, where the magnetic field is expected to be weaker and more horizontal (see Paper II).

The time-averaged values of brightness, size, and horizontal velocity of small-scale umbral features are completely uncorrelated. However, during the evolution of an individual feature, there is a correlation between the brightness excess $\Delta I$ with respect to the surrounding umbra and the area of the feature (Paper II). An example of the evolution of a long-lived CUD is shown in the left panel of Fig. 5. The correlation between $\Delta I$ and area was 0.68 in this case. Coefficients of correlation between $\Delta I$ and area evolution were calculated for the non-split/merge features (337 UDs and 114 bright features in faint LBs 2 and 3) and the histograms are plotted in the right panel of Fig. 5. The temporal brightness and size variations are positively correlated in most cases $-50 \%$ of features have correlation coefficients higher than 0.48 and the typical value of a correlation is 0.60 . The features first grow and brighten and then get smaller and fainter, in agreement with theoretical predictions (e.g. Schüssler \& Vögler $2006)$. For the other features that split or merge, the $\Delta I$-to-area correlation is much weaker but still detectable. The histograms (not shown here) are asymmetric with prevailing positive values and have flat maxima around 0.40 . For $50 \%$ of split/merge

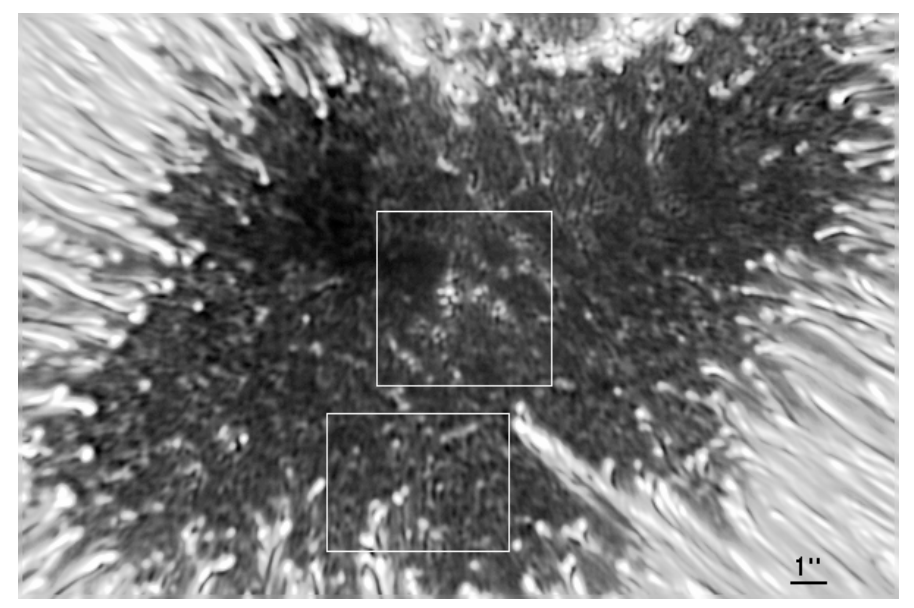

Fig. 6. MFBD reconstructed red-band image of the umbra taken at 08:59 UT. This image is enhanced by the unsharp masking technique for a better visualisation. The upper rectangle contains a group of central umbral dots with dark lanes, the lower one a field of peripheral umbral dots with tails.

features, the correlation coefficients are higher than 0.22 . The reason for the weak correlation is that the split/merge events introduce substantial uncorrelated changes in brightnesses and sizes of the features.

\subsection{Dark lanes in central umbral dots}

A group of bright (0.3-0.5 $\left.I_{\mathrm{ph}}\right)$, large ( 0. ' 4), and long-lived CUDs was present in the central part of the umbra from 08:30 to 10:05 UT (see Fig. 6, upper box). This group moved slowly in the direction of LB 2. Dark lanes, predicted by theoretical simulations (Schüssler \& Vögler 2006), are observed in several CUDs of this group. Obviously, because of the limited spatial resolution, we are able to observe dark lanes only in bright and large UDs. According to simulations, such UDs should show multifold dark lanes, separating them into several parts. In real observations, it is difficult to distinguish such UDs from conglomerates of unrelated small UDs. However, the integrity of the observed CUDs during the time period of about one hour indicates that we indeed see CUDs with dark lanes.

The 43 min series of MFBD reconstructed red-band frames from 08:43 to 09:26 UT with a temporal resolution $12.6 \mathrm{~s}$ is used to study the substructures in the four brightest CUDs in detail. Equivalent substructures are also seen in the reconstructed blue-band frames, but with lower signal-to-noise ratio. Threeor fourfold dark lanes separate CUDs into 3-4 parts. The substructures vary with time (the typical time scale is about $3 \mathrm{~min}$ ) and the dark lanes disappear and reappear during the evolution of CUDs. These changes may be caused by residual seeing variations.

The measurement of brightness, size, and temporal changes of four CUDs, their parts, and dark lanes was made in a series of segmented images of the field delimited by the upper box in Fig. 6. The segmentation was made in two steps. First, CUDs were separated from the background using the multilevel segmentation (Bovelet \& Wiehr 2001; Roudier 2003, private communication). Then the dark lanes and parts of CUDs were identified by means of the low-noise curvature determination algorithm (Hamedivafa 2008). An example of the original and segmented image is displayed in Fig. 7.

The basic observed parameters of CUDs 1-4 are summarised in Table 1 . The table contains the values of CUD peak intensity 


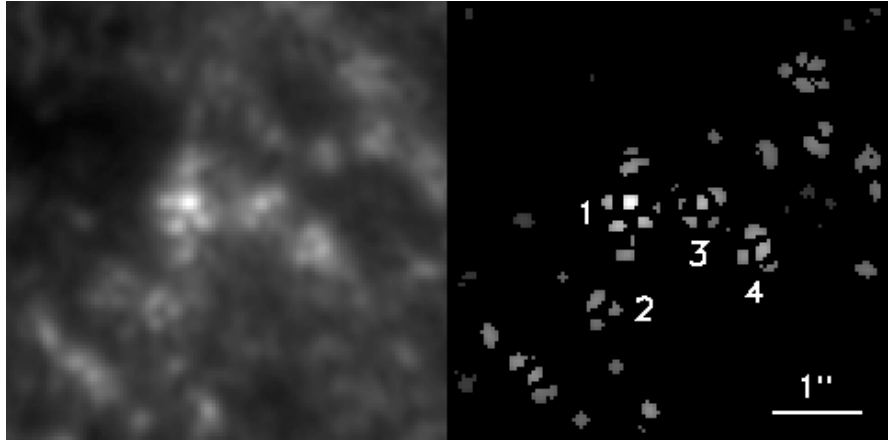

Fig. 7. Central umbral dots with dark lanes. The field corresponds to the upper box in Fig. 6. Left - original image, right - corresponding segmented image. Umbral dots under study are marked by numbers.

Table 1. Observed parameters of central umbral dots with dark lanes (see text for the explanation of symbols).

\begin{tabular}{ccccccc}
\hline \hline CUD & $I_{\mathrm{ud}}$ & $I_{\mathrm{b}}$ & $I_{\mathrm{dl}}$ & $d$ & $t[\mathrm{~min}]$ & $t_{\mathrm{dl}}[\mathrm{min}]$ \\
\hline 1 & 0.54 & 0.14 & 0.43 & 0.41 & 80 & 35 \\
2 & 0.36 & 0.15 & 0.28 & $0{ }^{\prime} 40$ & 75 & 10 \\
3 & 0.43 & 0.18 & 0.32 & 0.42 & 70 & 14 \\
4 & 0.43 & 0.16 & 0.37 & 0.44 & 50 & 14 \\
\hline
\end{tabular}

$I_{\mathrm{ud}}$, intensity $I_{\mathrm{b}}$ of the surrounding umbral background, intensity $I_{\mathrm{dl}}$ of the dark lanes, and diameter $d$. All the intensities are in units of $I_{\mathrm{ph}}$. The values of intensities and diameters are the averages of measurements in four frames for each CUD showing the best-resolved substructure. The lifetime $t$ of CUDs is measured in the $6 \mathrm{~h} 23 \mathrm{~min}$ long time series and $t_{\mathrm{dl}}$ is the total time for which the dark lanes were observed during the 43 min long series of reconstructed frames. The peak-to-background ratio $I_{\mathrm{ud}} / I_{\mathrm{b}}$ for the brightest CUD 1 is 3.9 , for CUDs $2-4$ is approximately 2.5 . The average ratio $I_{\mathrm{dl}} / I_{\mathrm{ud}}$ is 0.8 . Since the dark lanes are very narrow, this value has to be considered as an upper limit.

The feature-tracking algorithm was applied to the $43 \mathrm{~min}$ series of segmented images to measure the lifetimes and sizes of the individual parts of CUDs, separated by the dark lanes. The mean and median lifetimes are 9 min and 4 min, respectively. The median value is comparable to the characteristic time of temporal variations of the substructures $(3 \mathrm{~min})$ estimated by the visual inspection of the movie. Residual seeing fluctuations may be the cause of these changes. Both the mean and median sizes of the parts of CUDs are 0'! 17 . The apparent width of the dark lanes measured in the segmented images is $0{ }^{\prime} 08-0$ ! $^{\prime} 12$ (2-3 pixels), i.e., smaller than our resolution limit 0 ' 14 . Nevertheless, because the dark lanes are easily detected in the restored images (see Fig. 7, left panel), we may expect that they are not much narrower than $0 . ' 14$.

\subsection{Tails of peripheral umbral dots}

In Paper I we mentioned that narrow bright or dark tails are often attached to PUDs. In the 43 min series of MFBD reconstructed red- and blue-band images, a well-resolved pattern of radially oriented narrow bright and dark filaments is observed in the peripheral parts of the umbra (see Fig. 6). These filaments are not connected to the penumbra. The rms contrast of the pattern is only $0.02-0.03 I_{\mathrm{ph}}$ in the red-band images. Most of the filaments are attached to PUDs, giving the appearance of comet-like tails pointing in the direction toward the penumbra (cf. Rimmele 2008). The presence of the attached filaments is

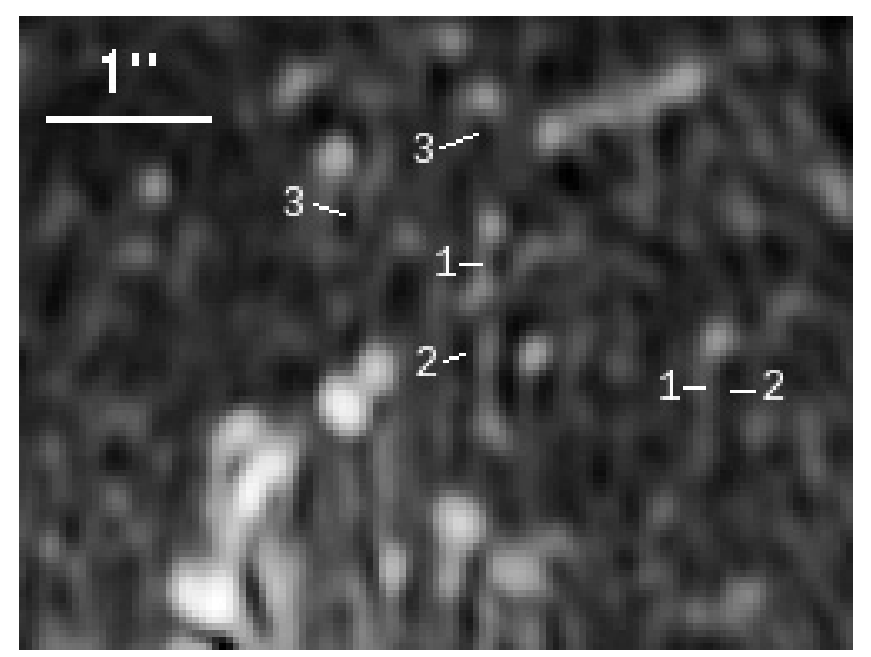

Fig. 8. Narrow bright and dark filaments (tails) connected to peripheral umbral dots. Numbers label examples of: 1 - bright filaments, 2 - dark filaments, 3 - tails composed of two bright and one dark filament. The image is enhanced by the unsharp masking technique for a better visualisation. The field corresponds to the lower box in Fig. 6 .

independent of the brightness of PUDs. An example is shown in Fig. 8, a magnification of the lower box in Fig. 6. The image is enhanced by unsharp masking for a better visualisation of the small-scale structure. Examples of bright and dark filaments are labelled by numbers 1 and 2 , respectively.

The basic observed parameters of the bright and dark filaments were measured in the best images of the reconstructed red-band series. The typical width of the filaments is at or below the resolution limit 0 !' 14 . Their length ranges from 0.44 to $1^{\prime \prime}$. The intensity of the bright filaments is approximately 0.06 $I_{\mathrm{ph}}$ higher than the intensity of the dark ones. The filaments live 5-20 min, what is comparable with the lifetime of UDs.

In many cases, two bright filaments with a dark one between them are attached to one PUD, forming a composite tail approximately 0.'4 wide, similar to a short dark-cored penumbral filament. Such dark-cored tails are seen in Fig. 8 and also in the right part of Fig. 6 at the periphery of the umbra and near the strong light bridge. Sometimes we observe that one bright filament is missing, probably because it is too faint and narrow to be detected, sometimes the dark-cored tails overlap. For this reason we expect that the dark-cored tails of PUDs are in fact more numerous than we observe. Such composite tails also appear as a result of the theoretical simulations of magnetoconvection in an inclined magnetic field. They are well visible in Fig. 1 of Rempel et al. (2009) at the periphery of the simulated umbra.

\section{Discussion and conclusions}

We analysed the $6 \mathrm{~h} 23 \mathrm{~min}$ time series of broadband images $(\lambda=602.0 \pm 1.3 \mathrm{~nm})$ of a large $\left(d_{\mathrm{eff}}=21^{\prime \prime}\right)$ and dark $\left(I_{\min }=0.09 I_{\mathrm{ph}}\right)$ umbra. A 43 min part of this series was reconstructed by means of the MFBD method (Löfdahl 2002) and used to study the substructures of UDs - dark lanes in CUDs and narrow tails attached to PUDs.

Two faint LBs with dark central channels (LB 2 and LB 3, see Fig. 1) were formed in the umbra. The formation phase took about $2 \mathrm{~h}$, followed by the phase of stability. Small (0'!17) bright dot-like features moved along these LBs. The dark central channels in faint LBs may have a similar physical origin like the dark lanes in UDs, i.e., the enhanced density at the top of ascending hot gas (Rimmele 2008; Giordano et al. 2008). Two 
bright penumbral filaments extending deep into the umbra developed subsequently at nearby positions ("4" in Fig. 1). Each one showed two phases of growth (50 and $30 \mathrm{~min})$, then a separation from the penumbra (20 min), and a final decay into several PUDs (30 min). The total lifetime of one penumbral filament was $3 \mathrm{~h}$.

Lifetimes, sizes, and horizontal velocities of UDs and dotlike features in faint LBs were measured using a feature tracking technique applied to the $117 \mathrm{~min}$ long best part of the unreconstructed time series. The majority $(90 \%)$ of all observed features split or merge with other features. The median lifetime of both types of features is approximately $3.5 \mathrm{~min}$ if the life of a feature is expected to finish when it splits or merges. If the split/merge events are not considered to be the end of life (according to the rules described in Sect. 3.2), the median lifetime is $5.7 \mathrm{~min}$. Both UDs and features in faint LBs that do not split or merge are clearly smaller $\left(0 .^{\prime} 15\right)$ than the average size $\left(0{ }^{\prime} 17\right)$ of all features. This means that features showing the split or merge events may be composed to a large extent of small unresolved objects. The obtained sizes are much smaller compared to 0.'31 reported by Riethmüller et al. (2008b). Horizontal motions of umbral bright small-scale features are directed either into the umbra or along faint LBs. Time-averaged velocity magnitudes $\left(0.34 \mathrm{~km} \mathrm{~s}^{-1}\right)$ are similar for UDs and features in faint LBs and do not depend on the split or merging of the features. Features faster than $0.4 \mathrm{~km} \mathrm{~s}^{-1}$ mostly appear at the periphery of the umbra and in faint LBs, where the magnetic field is expected to be weaker and more horizontal. Although the time-averaged values of brightness, size, and horizontal velocity of features are uncorrelated, during the evolution of individual non-split/merge features the temporal brightness variations are positively correlated with the size variations in most cases.

For the first time, we tried to measure the detailed photometric parameters of CUDs with dark lanes in the series of reconstructed images. For this purpose we selected a group of four bright, large, and long-lived CUDs in the centre of the umbra. They were brighter by a factor of 2.5 (3.9 in one case) than the surrounding background. The three- or fourfold dark lanes, observed with the resolution of 0. ' 14 , correspond to narrow - below the resolution limit $-20 \%$ deep depressions in the intensity profiles of CUDs. This result has to be taken with care, because of the strong contamination by stray light from the bright parts of CUDs. The substructures vary strongly with time. The median lifetime of the bright parts of CUDs is $4 \mathrm{~min}$, and the dark lanes disappear and reappear. It is a question of whether these changes, not seen in the simulations by Schüssler \& Vögler (2006), might be caused by the residual seeing variations in the reconstructed images. The average size and lifetime of the four selected CUDs with dark lanes (0!'42 and $70 \mathrm{~min})$ are comparable to the corresponding parameters of UDs appearing in the simulations $\left(0 .{ }^{\prime} 3-\right.$ $0{ }^{\prime} 4$ and $30 \mathrm{~min}$ ). However, such large and long-lived CUDs are not frequent in umbrae. The majority of UDs are smaller $\left(0 .{ }^{\prime} 2-\right.$ 0.3 ) and live much shorter time (3-9 min) - see also Riethmüller et al. (2008b). The future theoretical simulations should reproduce also such small and short-lived UDs.

We confirm the bright and dark filamentary tails connected to PUDs and directed toward the penumbra, reported in Paper I and by Rimmele (2008). In fact, such substructures are attached to nearly all PUDs, independent of their brightness. We newly observe that many tails of PUDs are composed of two narrow bright and one dark parallel filaments, forming a structure $0 . ' 4$ wide that resembles a short dark-cored penumbral filament. The length of this structure is $0{ }^{\prime} 4-1^{\prime \prime}$. The lifetime of such darkcored tails is comparable to the lifetime of UDs. It can be expected that the origin of central dark filaments in the tails is analogous to the origin of the dark lanes in UDs and dark cores in penumbral filaments. Sobotka \& Jurčák (2009) found that, concerning the physical characteristics, PUDs are similar to PGs located at the tips of bright penumbral filaments. The discovery of the dark-cored tails attached to PUDs strongly supports this similarity. Such structures also appear as a result of the radiative MHD simulation of a sunspot by Rempel et al. (2009). These authors claim that the underlying processes for the formation of penumbral filaments are the same as those for umbral dots. From this point of view, PUDs with dark-cored tails result from magnetoconvective energy transport in the form of hot rising plumes inside the inclined magnetic field at the periphery of the umbra. They represent a transition from CUDs to PGs and bright penumbral filaments.

We conclude that the numerical MHD simulations of magnetoconvection in the sunspot umbra by Schüssler \& Vögler (2006) and Rempel et al. (2009) correctly predict and reproduce the observed substructures - dark lanes in CUDs and tails attached to PUDs.

Acknowledgements. We express our thanks to C. Möstl, R. Kever, and R. Henderson for assisting in the observations. We are grateful to J. A. Bonet and S. Vargas Domínguez for helping us to get familiar with the MFBD algorithm, and to A. de Vicente for his support on the Condor workload management system (http://www.cs.wisc.edu/condor/). The authors thankfully acknowledge the technical expertise and assistance provided by the Spanish Supercomputing Network (Red Española de Supercomputación), as well as the computer resources - the La Palma Supercomputer, located at the Instituto de Astrofísica de Canarias. This work was supported by grant IAA 300030808 of the Grant Agency of the Academy of Sciences of the Czech Republic (AS CR), by the Research Plan AVOZ 10030501 of AS CR, by the OPTICON TransNational Access Programme, and by the Spanish Ministerio de Educación y Ciencia through the project ESP 2006-13030-C06-01. The SST is operated by the Royal Swedish Academy of Sciences in the Spanish Observatorio del Roque de los Muchachos of the Instituto de Astrofísica de Canarias.

\section{References}

Bharti, L., Jain, R., \& Jaafrey, S. N. A. 2007a, ApJ, 665, L79 Bharti, L., Joshi, C., \& Jaafrey, S. N. A. 2007b, ApJ, 669, L57

Bharti, L., Joshi, C., Jaafrey, S. N. A., \& Jain, R. 2009, MNRAS, 393, 65

Bovelet, B., \& Wiehr, E. 2001, Sol. Phys., 201, 13

Choudhuri, A. R. 1986, ApJ, 302, 809

Giordano, S., Berrilli, F., Del Moro, D., \& Penza, V. 2008, A\&A, 489, 747

Grossmann-Doerth, U., Schmidt, W., \& Schröter, E. H. 1986, A\&A, 156, 347

Hamedivafa, H. 2008, Sol. Phys., 250, 17

Kitai, R., Watanabe, H., Nakamura, T., et al. 2007, PASJ, 59, S585

Löfdahl, M. G. 2002, Proc. SPIE, 4792, 146

Martínez Pillet, V. 1992, Sol. Phys., 140, 207

November, L. J., \& Simon, G. W. 1988, ApJ, 333, 427

Parker, E. N. 1979, ApJ, 234, 333

Rempel, M., Schüssler, M., \& Knölker, M. 2009, ApJ, 691, 640

Riethmüller, T. L., Solanki, \& Lagg, A. 2008a, ApJ, 678, L157

Riethmüller, T. L., Solanki, S. K., Zakharov, V., \& Gandorfer, A. 2008b, A\&A, 492,233

Rimmele, T. 2008, ApJ, 672, 684

Scharmer, G. B., Bjelksjö, K., Korhonen, et al. 2003, in Innovative Telescopes and Instrumentation for Solar Astrophysics, ed. S. Keil, \& S. Avakyan, Proc. SPIE, 4853, 341

Schüssler, M., \& Vögler, A. 2006, ApJ, 641, L73

Sobotka, M., \& Jurčák, J. 2009, ApJ, 694, 1080

Sobotka, M., \& Puschmann, K. G. 2007, in Modern Solar Facilities Advanced Solar Science, ed. F. Kneer, K. G. Puschmann, \& A. D. Wittmann (Universitätsverlag Göttingen), 205

Sobotka, M., Brandt, P. N., \& Simon, G. W. 1997, A\&A, 328, 682

Sobotka, M., Puschmann, K. G., \& Hamedivafa, H. 2008, Centr. Eur. Astrophys. Bull., 32, 125

Thomas, J. H., \& Weiss, N. O. 2008, Sunspots and Starspots (Cambridge University Press)

Weiss, N. O., Brownjohn, D. P., Hurlburt, N. E., \& Proctor, M. R. E. 1990, MNRAS, 245, 434 